\title{
Decreased Sun-Earth energy-coupling efficiency starting from 2006
}

\author{
Masatoshi Yamauchi
}

\begin{abstract}
The geoeffect of extremely low solar (sunspot) activity starting from the last solar minimum is one of the major space science issues. The present study examines long-term changes of geomagnetic responses seen as Dst, Kp, and AL indices to the same solar wind conditions (density, velocity, magnetic field, and their products) using the NASA OMNI hourly values up to August 2014. Both decadal averages (1965 to 1974, 1975 to 1984, 1985 to 1994, 1995 to 2004 that approximately correspond to solar cycles \#20 to 23, respectively, and from 2005) and annual averages indicate that the geomagnetic activity for a given solar wind condition, namely the Sun-Earth coupling efficiency, decreased quantitatively from around 2006 until now compared to the previous four decades. The decrease remains even after the EUV flux (using F10.7 index) is considered and is more obvious in the low-latitude geomagnetic disturbances (Dst) than in the high-latitude geomagnetic disturbances (AL). The results cannot be explained by existing explanations including the ionospheric conductivity effect in the magnetosphere-ionosphere coupling system.
\end{abstract}

Keywords: Sun-Earth coupling; Solar wind energy input; OMNI hourly data; Dst; AL; Kp; Geomagnetic activity; Akasofu epsilon; Solar cycle 24

\section{Correspondence/findings \\ Background}

The sunspot cycle (the so-called 11-year solar cycle) varies from cycle to cycle over many decades in terms of its peak amplitude (e.g., peak sunspot number and number of X-type largest flares), average amplitude during the entire cycle (e.g., average coronal magnetic field and average solar wind velocity), length of the solar cycle, and the length and depth of the solar minimum defined by the sunspot numbers (Siscoe 1980; Feynman and Fougere 1984; Friis-Christensen and Lassen 1991; Usoskin et al. 2007; Svalgaard 2009; Abreu et al. 2012). Thus, the Sun and the corona show long-term variations (here the term 'long-term' means time scales longer than two solar cycles). For example, there was no sunspot maximum for nearly 70 years around the late 17th century (Maunder Minimum), but the 11-year cycle restarted and repeated for many centuries.

Such long-term solar variations may affect the Earth through different interplanetary parameters, such as the solar wind, heliospheric magnetic field, solar energetic

Correspondence: M.Yamauchi@irf.se

Swedish Institute of Space Physics, Kiruna, Box 812, Rymdcampus 1, S-98128 Kiruna, Sweden particles, solar extreme ultraviolet (EUV) flux, and solar radio bursts. In fact, the long-term geomagnetic activities and climate are correlated to the long-term solar variation (e.g., Friis-Christensen and Lassen 1991; Lockwood et al. 1999; Lockwood 2012; Svalgaard and Cliver 2005; Feynman and Ruzmaikin 2011; Stauning 2011; IPCC 2014). Friis-Christensen and Lassen (1991) found that centurial variation of the global temperature shows a similar profile as that of the solar cycle length until the mid 1980s before the anthropogenic effect became the major cause of rapid global warming (Stauning 2011; IPCC 2014). Lockwood (2012) showed that local climate is related to the long-term variation of the solar magnetic activity. Thus, the Sun-Earth coupling varies over different solar cycles.

On the other hand, short-term Sun-Earth coupling through the plasma and magnetic field has long been studied in the context of space weather. After Akasofu and his co-worker succeeded in quantitatively predicting instantaneous (less than 1-h resolution) geomagnetic activity from the solar wind input (Perreault and Akasofu 1978; Akasofu 1981), many works have been developed to refine it in obtaining the Sun-Earth coupling function (e.g., Newell et al. 2007; Svalgaard 2009). This attempt also

\section{是 Springer}

(c) 2015 Yamauchi; licensee Springer. This is an Open Access article distributed under the terms of the Creative Commons Attribution License (http://creativecommons.org/licenses/by/4.0), which permits unrestricted use, distribution, and reproduction in any medium, provided the original work is properly credited. 
includes solar parameters such as the F10.7 flux (proxy of the solar EUV) as the input parameters. However, very little work has considered the variation of the Sun-Earth coupling function itself.

In this context, Feynman and Fougere (1984) showed that the geomagnetic activity at mid-latitude Greenwich and Melbourne during the solar minimum is different between different solar cycles with similar sunspot numbers. The result suggests that the Sun-Earth coupling function (or its coefficient) may be a function of the strength of the solar cycle. The most recent solar minimum (year 2009) was a good example that demonstrated the cycle-to-cycle difference. Pulkkinen et al. (2011, 2014) showed that the auroral location and the AL index during 2009 are different from those of the previous solar minimum (1996) for the same solar wind conditions. The difference between these two solar minima (1996 and 2009) is also seen in the global ionospheric density (Solomon et al. 2013), which was simply explained by the difference in the solar EUV intensity between these two minima (see also Bergeot et al. 2013). The relation between the solar EUV and the location of the current system is also examined by Ohtani et al. (2014). Using satellite data to obtain the field-aligned current, they showed that the change in the location of the magnetosphere-ionosphere (M-I) current system depends on the F10.7 index and suggested that this could be the cause of the low Sun-Earth coupling during 2009.

If the shift of M-I current system with low ionospheric conductivity is the major reason for the uniqueness of year 2009, the effect should be more obvious in the highlatitude geomagnetic disturbances (AL index) than in the low-latitude geomagnetic disturbances (Dst index). Also, the uniqueness should not be limited to the solar minimum because the solar EUV of the current solar cycle is lower than the previous cycles. To examine whether these expectations are correct or not, the Sun-Earth coupling should be systematically compared at all latitudes and for the entire solar cycle to the other solar cycles. The purpose of this paper is to examine them using the geomagnetic indices (Dst, $\mathrm{Kp}$, and $\mathrm{AL}$ representing equatorial, mid-latitude, and auroral latitudes, respectively) and the measured solar wind data (NASA OMNI data).

\section{Methods}

Ten-year average values of geomagnetic (a) hourly Dst, (b) $\mathrm{Kp}$ (Kp is a 3-h index), and (c) hourly AL indices are obtained against different values of the solar wind parameters, F10.7 index, and their products. The raw solar wind parameters include the proton density $\mathrm{N}_{P}$, proton velocity $\mathrm{V}_{S W}$, dynamic pressure $\mathrm{P}_{S W}$, interplanetary magnetic field $\mathrm{B}$, its northward component $\mathrm{B}_{Z}$, and tangential component $\mathrm{B}_{\tan }=\sqrt{\mathrm{B}_{Y}^{2}+\mathrm{B}_{Z}^{2}}$. The products of the solar wind parameters include the duskward electric field $\mathrm{E}_{Y}=-\mathrm{V}_{S W} \cdot \mathrm{B}_{Z}$, mapped electric field $\mathrm{E}_{K L}=\mathrm{V}_{S W} \cdot \mathrm{B}_{\tan } \cdot \sin ^{2}\left(\theta_{\mathrm{c}} / 2\right)$ (Kan and Lee 1979), modified Akasofu's energy-coupling functions $\epsilon^{\prime}=\left(4 \pi / \mu_{0}\right) \cdot V_{S W} \cdot B_{\text {tan }}^{2} \cdot \sin ^{4}\left(\theta_{c} / 2\right)$ (Perreault and Akasofu 1978; Akasofu 1981), and the flux accumulation rate in the magnetosphere $d \Phi / d t=\left(\mathrm{V}_{S W}^{2} \cdot \mathrm{B}_{\tan } \cdot \sin ^{4}\left(\theta_{\mathrm{c}} / 2\right)\right)^{2 / 3}$ (Newell et al. 2007), where $\cos \left(\theta_{\mathrm{c}}\right)=\mathrm{B}_{\mathrm{Z}} / \mathrm{B}_{\tan }$. For $\epsilon^{\prime}, \mathrm{B}_{\tan }$ that represents anti-sunward energy flux is used instead of $B$ of the original form of $\epsilon$ (dash is added to differentiate, but the result is almost the same), and the constant coefficient (e.g., contact length at the magnetopause $l_{0}=7$ to $10 \mathrm{R}_{E}$ ) is not included. All data are obtained through the NASA OMNI database. This database contains data from 1962 , but the data coverage of the first 3 years is relatively poor, and therefore, data from 1965 was used.

While the raw solar wind parameters and electric field are scaled linearly, the energy-coupling function $\left(\epsilon^{\prime}\right)$, the flux accumulation rate $(d \Phi / d t)$, and the mapped electric field $\left(E_{K L}\right)$ are scaled (binned and averaged) in logarithmic scales (this makes the number of data more equally distributed) because the present purpose is not examining the coupling function but to visualize the major differences in average, if any, between the most recent 10 years and the previous decades. For the same reason, hourly resolution data was used, and the past 50 years data are simply divided by every 10 years, 1965 to 1974 (approximately cycle \#20), 1975 to 1984 (approximately cycle \#21), 1985 to 1994 (approximately cycle \#22), 1995 to 2004 (approximately cycle \#23), and 2005 to 2014 (from after the declining phase of cycle \#23 to the maximum of cycle \#24). In addition to 10-year averages, bi-annual averages (not shown here) and annual averages are also examined to confirm that the result does not depend on the averaging scheme.

\section{Results}

Figure 1 shows the average values of geomagnetic (a) Dst, (b) $\mathrm{Kp}$, and (c) AL indices that are plotted against the modified Akasofu's energy-coupling function $\epsilon^{\prime}$. For most of the $\epsilon^{\prime}$ bins $\left(\epsilon^{\prime}<10^{2} \mathrm{~W} / \mathrm{km}^{2}\right.$, which covers $>90 \%$ of hours), the standard deviations $\sigma$ are about 10 to $20 \mathrm{nT}$ for Dst, 1 for Kp, and $70 \%$ to $130 \%$ for AL, i.e., widely distributed. Yet, the average geomagnetic activities (absolute values of Dst, Kp, and $\mathrm{AL}$ ) for the same $\epsilon^{\prime}$ values (when $\epsilon^{\prime}<10^{2} \mathrm{~W} / \mathrm{km}^{2}$ ) are systematically smaller during the most recent 10 years (after the declining phase of the cycle \#23) than the previous four decades (cycles \#20 to 23) beyond the estimated statistical error for all three indices.

Note that Kp is nonlinearly scaled, but the speciality of the most recent 10 years is not an artifact of such nonlinear averaging. In fact, the probability of having $\mathrm{Kp} \geq$ 4 in Figure $1 \mathrm{~d}$ demonstrates that the chances of high-Kp activities for the same $\epsilon^{\prime}$ values are much lower during the 
(a) hourly Dst [nT]

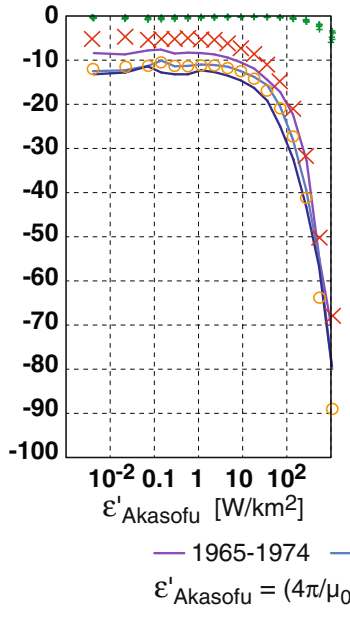

(b) $\mathrm{Kp}(+=0.3,-=-0.3)$

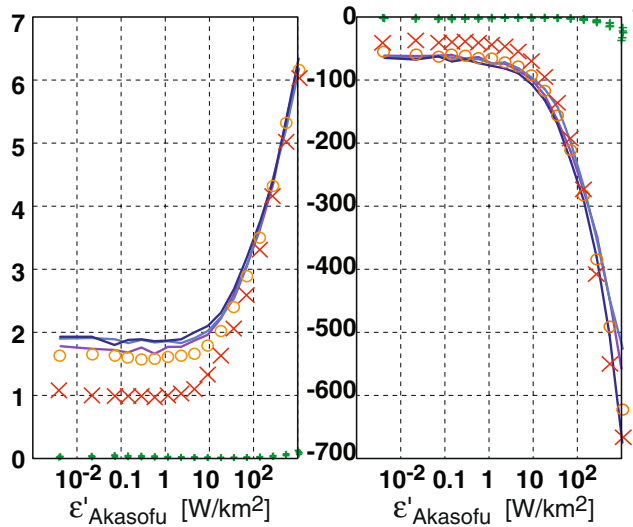

(c) hourly AL [nT]

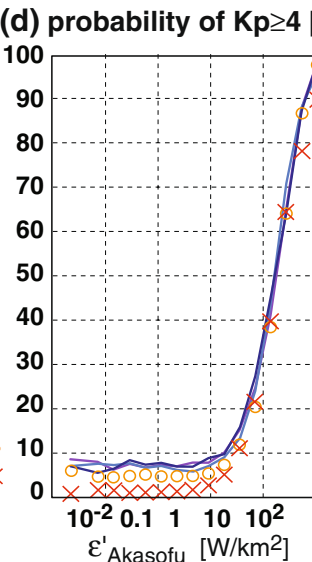

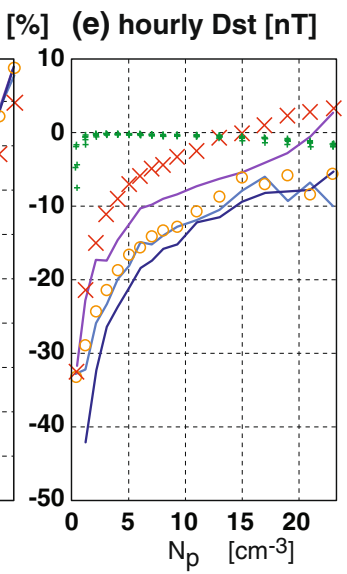

EAkasofu

$\left.\mu_{0}\right) \cdot V^{*} \mathrm{BT}^{2} \sin ^{4}\left(\theta_{\mathrm{C}} / 2\right)=\left(4 \pi / \mu_{0}\right) \cdot \mathrm{V}^{*}(\mathrm{BT}-\mathrm{Bz})^{2} / 4\left[0.01 \mathrm{~W} / \mathrm{km}^{2}\right]($ when $\mathrm{km} / \mathrm{s}$ and $\mathrm{nT}$ and are used for $\mathrm{V}$ and $\left.\mathrm{B})\right]$

Figure 1 Decade averages of geomagnetic responses to the solar wind input. Hourly values of geomagnetic (a) Dst, (b) $\mathrm{Kp}$, and (c) AL indices, and (d) probability of $K p \geq 4$ are averaged and plotted against the modified Akasofu's epsilon $\epsilon^{\prime}=\left(4 \pi / \mu_{0}\right) \cdot V_{S W} \cdot B_{\text {tan }}^{2} \cdot \sin { }^{4}\left(\theta_{c} / 2\right)=\left(4 \pi / \mu_{0}\right)$. $V_{S W} \cdot\left(B_{\tan }-B_{Z}\right)^{2} / 4$. The total energy input is estimated by multiplying square of $I_{0}=7$ to $10 R_{E}$, i.e., about 3 to $5 \times 10^{9} \mathrm{~km}{ }^{2}$. Data are divided into five 10-year periods as indicated by the legend (purple lines, blue lines, dark blue lines, orange circles, and red crosses, which approximately correspond to solar cycles \#20 to 24, respectively). Green pluses near the horizontal axis denote the estimated statistical errors $(\sigma / \sqrt{(n-1)}$ where $n$ is the number of data point and $\sigma$ is the standard deviation). Representative values for each $\epsilon^{\prime}$ bin is obtained by logarithmic averaging. (e) Hourly values of geomagnetic Dst index are averaged and plotted against the solar wind proton density $\mathrm{N}_{p}$.

most recent 10 years compared to each of the previous four decades (less than half when $\epsilon^{\prime}<10^{2} \mathrm{~W} / \mathrm{km}^{2}$ ). The same results are also obtained for the probability of large |Dst $\mid$ or $|\mathrm{AL}|$ values (not shown here). In other words, the occurrence rate of magnetic storms and substorms for the same solar wind energy input has significantly decreased during the recent years.

Average Dst values versus the solar wind density $\mathrm{N}_{P}$ are also plotted in Figure 1e because Dst (when $>-10 \mathrm{nT}$ ) is the most sensitive to $\mathrm{N}_{P}$ among the solar wind parameters. Again, the average Dst for given $\mathrm{N}_{P}$ values when $\mathrm{N}_{P}>2 \mathrm{~cm}^{-3}$ is systematically higher (more positive side) during the most recent 10 years than the previous four decades (1965-2004).

To estimate the degree of reduction of the geomagnetic activity for the same solar wind conditions, relative values of the most recent 10 years (2005-2014 average) to the averages of the previous four decades (10-year averages are further averaged) are plotted in Figure 2a,c. Here, an offset of $+5 \mathrm{nT}$ is introduced when taking the ratio for the average Dst values because Dst becomes positive when the solar wind density is very large as shown in Figure 1e. Figure 2a demonstrates that when $\epsilon^{\prime}<10^{2} \mathrm{~W} / \mathrm{km}^{2}$, the average values of all three indices for the same $\epsilon^{\prime}$ are statistically smaller in amplitude (absolute value) during the most recent 10 years $(2005-2014)$ than the previous 40 years (1965-2004), and this difference is more drastic for smaller $\epsilon^{\prime}$ values with a saturated value of about $60 \%$ for $\epsilon^{\prime}<10 \mathrm{~W} / \mathrm{km}^{2}$ (which covers $>50 \%$ of hours).
The result indicates that the global geomagnetic activities for the same solar wind energy input to the magnetosphere are less during the most recent 10 years (approximately the solar cycle \#24) than those during the previous four decades. This contrasts small differences among the previous four 10-year periods (approximately cycles \#20, \#21, \#22, and \#23): the variation over these four 10-year periods is at about the statistical error range, with only two exceptions (Dst values during 1965 to 1974 (cycle \#20) and Kp values during 1995 to 2004 (cycle \#23) for given $\epsilon^{\prime}$ values fall between the values of cycle \#24 and the other cycles beyond the statistical error range).

The same results as Figure 1 are obtained if one uses Newell's flux accumulation rate $d \Phi / d t$ (Newell et al. 2007) as the input parameter. Instead of repeating the Figure 1 format, relative values of the most recent 10 years (2005-2014) compared to the averages of the previous four decades (approximately cycles \#20 to 23) are plotted in Figure 2b. The plot is very similar to that of Figure 2a: absolute values of averages of all three indices for the same $d \Phi / d t$ are statistically smaller during the most recent 10 years $(2005-2014)$ than the previous 40 years $(1965-2004)$ when $d \Phi / d t<10^{4}(\mu \mathrm{V} / \mathrm{m})^{2 / 3}$ that covers the majority of hours, and this difference is more drastic for smaller $d \Phi / d t$ values. The similar results are also obtained if one uses $\mathrm{E}_{K L}=\mathrm{V}_{S W} \cdot \mathrm{B}_{\tan } \cdot \sin ^{2}\left(\theta_{\mathrm{c}} / 2\right)$ as the input parameter.

The above input parameters are functions of solar wind velocity and magnetic field without contributions from 


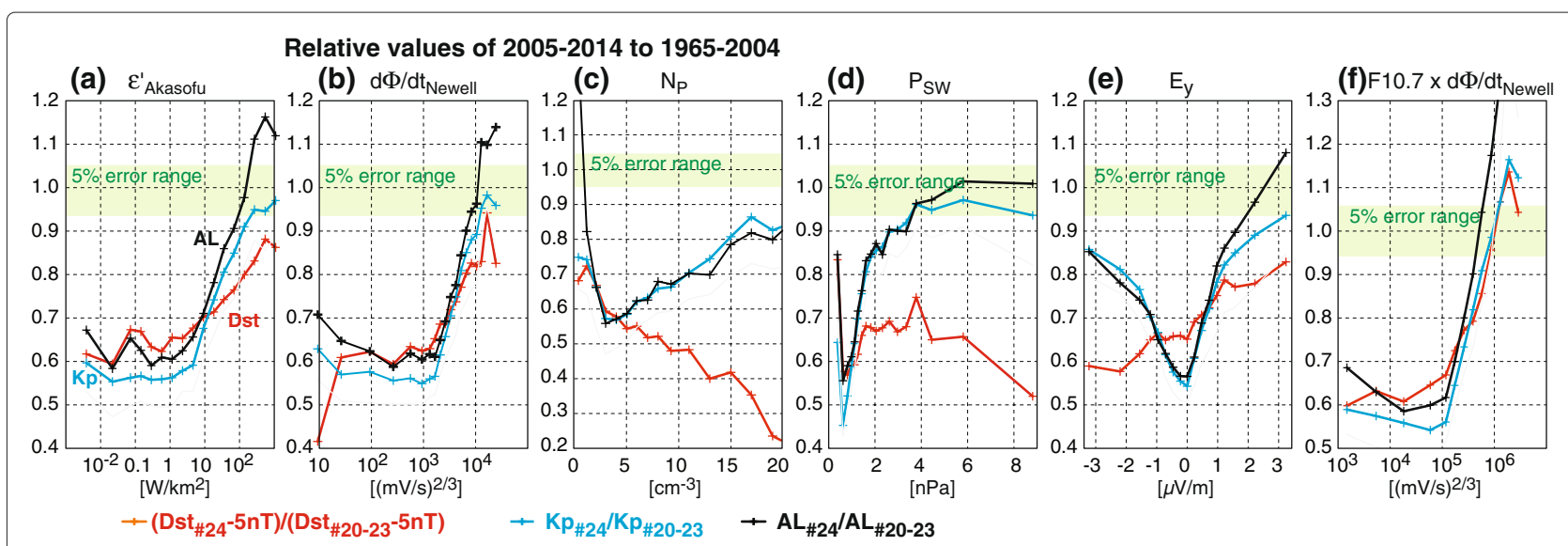

Figure 2 Relative efficiency of recent 10 year's Sun-Earth coupling compared to previous $\mathbf{4 0}$ years. Ratio of Dst, Kp, and AL values during the most recent 10 years (2005-2014) to the average of the previous four decades (10-year averages are further averaged) are plotted against various solar wind input parameters: (a) modified Akasofu's $\epsilon^{\prime}=\left(4 \pi / \mu_{0}\right) \cdot V_{S W} \cdot B_{\tan }^{2} \cdot \sin ^{4}\left(\theta_{c} / 2\right)$, (b) Newell's flux accumulation rate

$d \Phi / d t=\left(V_{S W}^{2} \cdot B_{\tan } \cdot \sin ^{4}\left(\theta_{c} / 2\right)\right)^{2 / 3}$, (c) solar wind proton density $N_{p}$, (d) solar wind dynamic pressure $P_{S W}$, (e) solar wind electric field $E_{Y}$, and (f) product of $d \Phi / d t$ and F10.7 index. The approximate error range (several \%) that includes both statistical error and the differences between each decade before 2005 is indicated by green hatch. Linear scales are used for $\mathrm{P}_{S W}, \mathrm{E}_{Y}$, and $\mathrm{N}_{P}$ while logarithmic scales are used for $\epsilon^{\prime}$ and $d \Phi / d t$.

$\mathrm{N}_{P}$ or $\mathrm{P}_{S W}$. Therefore, $\mathrm{P}_{S W}$ is also examined as the input parameter although it does not give as good a prediction as $\epsilon^{\prime}$ or $d \Phi / d t$. The relative values of the most recent 10 years compared to the previous four decades (approximately cycles \#20 to 23) in Figure 2d again demonstrate the decreased geomagnetic activities for the same solar wind dynamic pressure during the most recent 10 years when $\mathrm{P}_{S W}<4 \mathrm{nPa}$ (about $90 \%$ of hours).

The decreased geomagnetic responses to the same solar wind conditions $\left(\epsilon^{\prime}, \mathrm{d} \Phi / \mathrm{dt}, \mathrm{E}_{K L}\right.$, and $\left.\mathrm{N}_{P}\right)$ are valid for more than $90 \%$ of the time and are more prominent for smaller $\epsilon^{\prime}, \mathrm{d} \Phi / \mathrm{dt}$, or $\mathrm{E}_{K L}$ and for larger $\mathrm{N}_{P}$. Since small $\epsilon^{\prime}$ values generally mean northward interplanetary magnetic field (IMF), one may question how much this result is valid during southward IMF conditions that constitute the majority of large $\epsilon^{\prime}$ cases. To examine this, correlation with solar wind electric field $\mathrm{E}_{Y}$ is taken (Figure $2 \mathrm{e}$ ).

As expected, the ratio is asymmetric between positive $\mathrm{E}_{Y}$ (southward IMF) and negative $\mathrm{E}_{Y}$ (northward IMF). Yet, all values in Figure 2e are substantially less than unity as long as $\mathrm{E}_{Y}<+2 \mu \mathrm{V} / \mathrm{m}$ that covers the majority of hours. The asymmetry also suggests that the results of Figures 1 and 2 are valid for both northward and southward IMF (IMF direction sometimes changes within 1 $\mathrm{h})$. The other solar wind parameters such as $\mathrm{V}_{S W}, \mathrm{~B}, \mathrm{~B}_{Z}$, $\mathrm{V}_{S W} \cdot \mathrm{B}$, and $\epsilon^{\prime} \cdot \mathrm{N}_{P}$ are also examined (taken as horizontal axis of Figures 1 and 2, but not shown here), and the decreased geomagnetic activities for the same solar wind conditions are again obtained for all of these parameters for the majority of hours.

The next question is to identify the timing when the weak Sun-Earth coupling (lower geomagnetic activities for the same solar wind input) started. To examine this, annual variation of average Dst, $\mathrm{Kp}$, and $\mathrm{AL}$ indices are obtained for fixed values of $\epsilon^{\prime}$ and $\mathrm{N}_{P}$ in Figure 3. To increase statistics, binning of $\epsilon^{\prime}$ and $\mathrm{N}_{P}$ is taken wider than those in Figure 1. These bin values are represented by different colors in the figure.

Since hourly values instead of minute values are used (i.e., accuracy of time lag is $1 \mathrm{~h}$ ), the coupling efficiency might vary within a solar cycle. In fact, Figure 3 demonstrates large annual fluctuations. However, the baseline values certainly changed between 2005 and 2006 for Dst and $\mathrm{Kp}$, after which the absolute values are constantly lower than those before 2005 beyond the fluctuation level as long as $\epsilon^{\prime}<10^{2} \mathrm{~W} / \mathrm{km}^{2}$. Thus, the results obtained in Figures 1 and 2 do not depend on the way of dividing the data (here, the data is simply divided by every 10 years). The continuously decreased activity after 2006 is also seen in AL for small $\epsilon^{\prime}$ although it is not as obvious as Dst and Kp.

In Figure 3a,e, the Dst values are discontinuously high in 1965 with positive values even for large $\epsilon^{\prime}$ or relatively small $\mathrm{N}_{P}$. This singularity could be due to different zero line for the Dst calculation compared to the other years. Since the data is taken 50 years ago and the solar wind database is very little before, Dst zero lines in 1965 cannot easily be re-examined. If the singularity of the 1965 Dst value is due to such error, the Dst difference between the 1965 to 1974 decade and the following three decades (1975 to 2004) in Figure 1a,e is significantly reduced.

\section{Discussion}

In Figure 3, there are years of high $\mathrm{AL}$ and $\mathrm{Kp}$ activities for the same solar wind input during the declining phase (1974, 1983, 1994, and 2003), while the current 


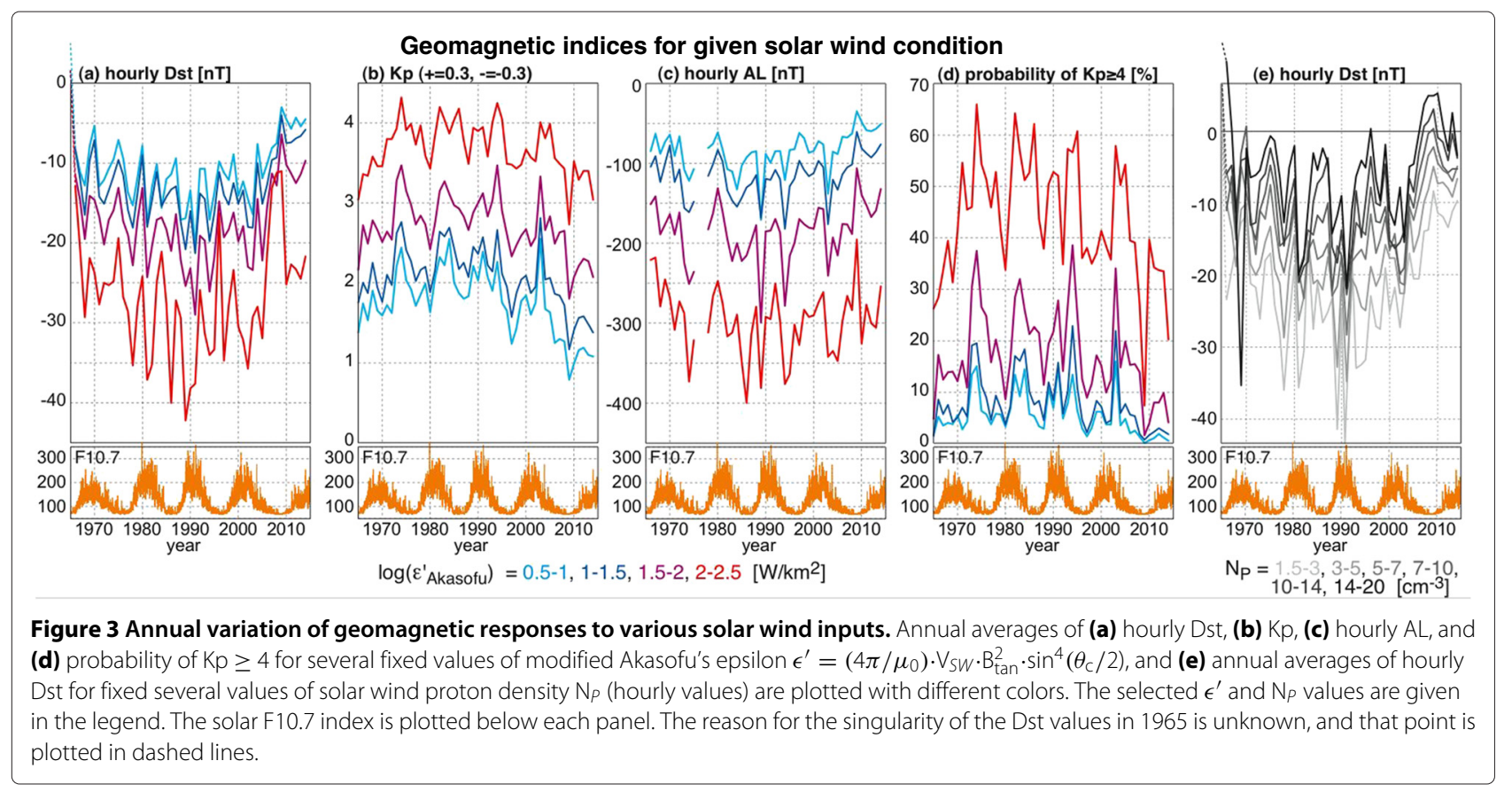

solar cycle data does not yet include such declining phase peaks. This means that the Figure 2 result could be exaggerated. Yet, the uniqueness of the recent years in the Sun-Earth coupling is still valid because the baseline in Figure 3 consistently changed from 2006. Therefore, the question is rather whether this speciality continues during the coming declining phase or not.

What causes the uniqueness of the recent years in the Sun-Earth coupling? Obvious candidates are ionospheric current and the M-I coupling system that are known to affect AL (Sato and Iijima 1979; Nagatsuma 2004; Ohtani et al. 2014).

Since the ionospheric conductivity controls them, we expect that the solar EUV flux (its F10.7 proxy) affects the AL response to the same solar wind conditions if the $\mathrm{M}-\mathrm{I}$ coupling is the major cause of the low geomagnetic response to solar wind input after 2006. The solar EUV flux also controls total amount of escaping ions from the ionosphere to the inner magnetosphere (Cully et al. 2003; Lundin et al. 2013), and hence might affect Dst through the change in the total amount of current carriers in the ring current region.

However, Figure 3 demonstrates that all Dst, Kp, and AL activities from 2011 when F10.7 values exceeded 100 (higher than values during past solar minima) are continuously lower than previous minima for the same solar wind energy input. Furthermore, the same analyses as Figures 1, 2 , and 3 for F10.7 index and its product with solar wind parameters (e.g., Figure 2f) suggest that the geomagnetic activity for the same external condition including the F10.7 index still decreased during the recent 10 years. Thus, the low solar EUV radiation is not the major driver of the low Sun-Earth coupling efficiency of the current solar cycle.

One should also note that the decrease of the Sun-Earth coupling is more drastic in Dst than AL, i.e., in the geomagnetic disturbances at locations away from the region 1 and 2 field-aligned current systems than those under these current systems. Physically, Dst mainly reflects ion drift motions in the inner magnetosphere and is much less related to the ionospheric conductivity or the M-I coupling system than AL.

These results stand against the hypothesis that the relatively low ionospheric conductivity and its effect on the M-I coupling system are the major cause of the low geomagnetic response to the solar wind input after 2006. The M-I coupling most likely plays some role, and actually, the shift of location of the auroral arc (and relevant MI coupling system) was detected during 2009 (Pulkkinen et al. 2011), but there must be other mechanism(s) that contributes to the low Sun-Earth coupling efficiency after 2006. The displacement of the ionospheric current cannot explain the drop of the Dst (Figure 3c). So far, the author cannot suggest a plausible mechanism to explain this.

On the other hand, the solar cycle-to-cycle difference and the recent low efficiency in the Sun-Earth coupling are debating topics for climatology as mentioned in the introduction. For examples, the global temperature is somewhat correlated to the strength of the solar cycle measured by the sunspot maximum or solar cycle length or average solar magnetic field over solar cycles (Friis-Christensen and Lassen 1991; Lockwood 2012; Stauning 2011), but the mechanisms that link such longterm activity and the climate have not been identified 
yet. It is possible that the unidentified mechanism in the present study and that in climatology might be related. In fact, Figure 3 shows that the trends of decadal variations in amplitudes are somewhat similar between the geomagnetic indices and solar F10.7 index.

Finally, a short note is given on high solar input cases (e.g., $\epsilon^{\prime}>10^{2} \mathrm{~W} / \mathrm{km}^{2}$ ). With a caution of relatively low statistics, Figure $2 \mathrm{a}$ indicates that AL response to the same solar wind energy input when $\epsilon^{\prime}>10^{2} \mathrm{~W} / \mathrm{km}^{2}$ is higher during the current solar cycle than the previous solar cycles. The same tendency is also seen in Figure 2b,e,f. In the past, solar cycle \#15 (1913 to 1923) was a very weak one but caused many hazardous magnetic storms, endorsing this tendency. If this is true (we need more statistics though), large coronal mass ejections during the coming declining phase have risks of hazardous consequences such as electrical failures.

\section{Conclusions}

Geomagnetic Dst, Kp, and AL indices for the same solar wind conditions (density, velocity, magnetic field, and their products), namely the Sun-Earth coupling efficiency, are significantly lower (in absolute values) during the most recent 10 years (2005 to 2014) than those during the previous four decades (1965 to 2004) according to the NASA OMNI hourly values up to August 2014. The decreased Sun-Earth coupling efficiency became obvious from around 2006 and has continued until now with a sharp drop in 2009. The decrease after 2006 is clearer in Dst than AL and is not an artifact of low ionospheric conductivity due to low solar EUV. The result cannot be explained by existing M-I coupling ideas alone but rather suggests that it might be related to the unidentified mechanism that connects the long-term solar activity and climate.

\section{Competing interests}

The author declares that he has no competing interests.

\section{Acknowledgements}

Dst, Kp, AL, and sunspot numbers (RI) are official IAGA- and IAA-endorsed indices that are provided by the World Data Center for Geomagnetism, Kyoto University, Japan (Dst and AL), GFZ, Adolf-Schmidt-Observatory Niemegk, Germany (Kp), and the Royal Observatory of Belgium, Brussels (RI). Including these indices, all data in hourly values are obtained from NASA-GSFC/SPDF through OMNIWeb (http://omniweb.gsfc.nasa.gov/ow.html).

The author thanks J. Neumann who made preliminary plots for nearly all parameters that are examined here as part of a summer internship from the University of Cologne, Germany, to the Swedish Institute of Space Physics. The author also thanks C. Stolle and R. Lundin for valuable comments.

Original analysis using monthly RI from 1932 instead of solar wind parameters was rejected by Geophys. Res. Lett. in April 2014 for two reasons: Reviewer \#1 could not find the long-term variation of the response of the mid-latitude geomagnetic field to the solar wind, and Reviewer \#2 requested to use solar wind data before making any conclusion with analyses only with Rl, and the author sincerely thanks the two reviewers for these comments.

Received: 29 October 2014 Accepted: 11 March 2015

Published online: 28 March 2015

\section{References}

Abreu JA, Beer J, Ferriz-Mas A, McCracken KG, Steinhilber F (2012) Is there a planetary influence on solar activity? Astronomy Astrophys 548:A88. doi:11.1051/0004-6361/201219997

Akasofu S-I (1981) Energy coupling between the solar wind and the magnetosphere. Space Sci Rev 28(2):121-190

Bergeot N, Tsagouri I, Bruyninx C, Legrand J, Chevalier JM, Defraigne P, Baire Q, Pottiaux E (2013) The influence of space weather on ionospheric total electron content during the 23rd solar cycle. J Space Weather Space Clim 3:A25. doi:10.1051/swsc/2013047

Cully CM, Donovan EF, Yau AW, Arkos GG (2003) Akebono/Suprathermal Mass Spectrometer observations of low-energy ion outflow: dependence on magnetic activity and solar wind conditions. J Geophys Res 108(A2):1093. doi:10.1029/2001JA009200

Feynman J, Fougere P (1984) Eighty-eight year cycle in solar terrestrial phenomena confirmed. J Geophys Res 89:302-3027

Feynman J, Ruzmaikin A (2011) The Suns strange behavior: Maunder Minimum or Gleissberg cycle? Solar Phys 272:351-363. doi:10.1007/s11207-011-9828-0

Friis-Christensen E, Lassen K (1991) Length of the solar cycle: an indicator of solar activity closely associated with climate. Science 254(5032):698-700

IPCC (2014) Working group I contribution to the IPCC fifth assessment report climate change 2013: the physical science basis. The Intergovernmental Panel on Climate Change. http://www.ipcc.ch/report/ar5/wg1/

Kan JR, Lee LC (1979) Energy coupling and the solar wind dynamo. Geophys Res Lett 6:577

Lockwood M, Stamper MR, Wild MN (1999) A doubling of the Sun's coronal magnetic field during the past 100 years. Nature 399:437-439

Lockwood M (2012) Solar influence on global and regional climate. Surv Geophys 33(3):503-534. doi:10.1007/s10712-012-9181-3.

Lundin R, Barabash S, Holmstrom M, Nilsson H, Futaana Y, Ramstad R, Mamauchi M, Dubinin E, Fraenz M (2013) Solar cycle effects on the ion escape from Mars. Geophys Res Lett 40(23):6028-6032. doi:10.1002/2013GL058154

Nagatsuma T (2004) Conductivity dependence of cross-polar potential saturation. J Geophys Res 109:A4. doi:10.1029/2003JA010286

Newell PT, Sotirelis T, Liou K, Meng C-I, Rich FJ (2007) A nearly universal solar wind-magnetosphere coupling function inferred from 10 magnetospheric state variables. J Geophys Res 112:A01206. doi:10.1029/2006JA012015

Ohtani S, Wing S, Merkin VG, Higuchi T (2014) Solar cycle dependence of nightside field-aligned currents: effects of dayside ionospheric conductivity on the solar wind-magnetosphere-ionosphere coupling. J Geophys Res 119:322-334. doi:10.1002/2013JA019410

Perreault WK, Akasofu S-I (1978) A study of geomagnetic storms. Geophys J R Astron Soc 54:547

Pulkkinen TI, Tanskanen El, Viljanen A, Partamies N, Kauristie K (2011) Auroral electrojets during deep solar minimum at the end of solar cycle 23. J Geophys Res 116:A04207. doi:10.1029/2010JA016098

Pulkkinen TI, Partamies N, Kilpua EKJ (2014) Substorm occurrence during quiet solar wind driving. J Geophys Res 119:2978-2989. doi:10.1002/2013JA019503

Sato T, lijima T (1979) Primary sources of large-scale Birkeland current. Space Sci Rev 24:347-366

Siscoe GL (1980) Evidence in the auroral record for secular solar variability. Rev Geophys Space Phys 18:647-658

Solomon SC, Qian L, and Alan G. Burns AG (2013) The anomalous ionosphere between solar cycles 23 and 24. J Geophys Res 118:6524-6535. doi:10.1002/jgra.50561

Stauning P (2011) Solar activity-climate relations: a different approach. J Atmos Solar-Terrest Phys 73(13):1999-2012. doi:10.1016/j.jastp.2011.06.011

Svalgaard L, Cliver EW (2005) The IDV index: its derivation and use in inferring long-term variations of the interplanetary magnetic field strength. J Geophys Res 110:A12103

Svalgaard L (2009) Observatory data: A 170-year Sun-Earth connection. In: Proceedings of the XIIIth IAGA workshop on geomagnetic observatory insturuments, data acquisition, and processing. pp 246-257

Usoskin IG, Solanki SK, Kovaltsov GA (2007) Grand minima and maxima of solar activity: new observational constraints. Astron Astrophys 471:301-309 